\title{
EVALUACIÓN DE FACTORES DE RIESGOS ASOCIADOS AL MANEJO MANUAL DE CARGA
}

\author{
Risk Factors Assessment associated to the Manual Load Handling
}

\author{
Lorena Martínez Sanhueza*
}

\section{RESUMEN}

La Ley $\mathrm{N}^{\circ}$ 20.001, del Libro II del Código del Trabajo de Chile del año 2005 define: que el peso máximo de carga humana es de $50 \mathrm{Kg}$., la obligatoriedad por parte del empleador de incorporar los medios mecánicos necesarios que eviten la manipulación manual de carga, y por último la capacitación a los trabajadores en esta materia.

La Escuela de Terapia Ocupacional de la Universidad Mayor, realiza el año 2007, en una empresa metalúrgica ligada a la minería metálica, un diagnóstico respecto de factores de riesgo asociados al manejo manual de carga en las áreas productivas y de servicios en dicha empresa, y así dar cumplimiento a la legislación, conocer con mayor precisión las condiciones reales del manejo manual de carga dentro de la empresa y su posible impacto en la adquisición de lesiones o enfermedades músculo esqueléticas en los trabajadores.

Para ello se utilizan dos metodologías de evaluación, el análisis de puesto de trabajo y el software Ergocargas, combinando estrategias de carácter cualitativo y cuantitativo respectivamente. Como parte de sus principales resultados se identifica que la conducta de trabajadores y la organización del trabajo constituyen aquellos factores de riesgo más relevantes. Por otra parte se destaca la importancia de abordar esta temática dentro de la empresa de manera trasversal entre los diversos departamentos que la componen es imprescindible para garantizar el control de los factores de riesgos asociados.

Palabras claves: Manejo Manual de Carga, Factores de Riesgo, Análisis de Puesto de Trabajo

\begin{abstract}
ABSTRAC
The Law No. 20001, at Book II of the Labor Code of Chile (2005) defines that the maximum load is $50 \mathrm{~kg}$ for human handling, and explain the obligation of the employer to incorporate the mechanical tools needed to avoid manual handling load, and finally the training of workers in this field. The School of Occupational Therapy, at Universidad Mayor, develops in 2007 at a company linked to the metalworking metal mining, a diagnosis about risk factors associated with manual handling of loads in the areas of production and services in the company, and to comply with the legislation, known more accurately the real conditions of the manual handling of heavy loads within the company and its possible impact on the acquisition of skeletal muscle injury or

\footnotetext{
* Terapeuta Ocupacional, Licenciada en Ciencias de la Ocupación Humana, Docente Escuela de Terapia Ocupacional Universidad Mayor Santiago. Camino La Pirámide 5750 - Huechuraba - Santiago de Chile. 56-2-3281405; lorena.martinez@umayor.cl
} 
disease in workers. There were used two methods for assessment, analysis of job placement and the software Ergocargas, strategies combining qualitative and quantitative respectively. As part of its main findings are that identifies the behavior of workers and work organization risk factors are those most relevant. On the other hand, it is seems that a critical point is the importance of addressing this issue within the company on a cross between the various departments that comprise it is essential to ensure control of risk factors associated.

Key words Charge Handling Management, Risk Factors, Work Position Analysis.

\section{INTRODUCCION}

La prevención de riesgos adquiere cada día mayor relevancia dentro de las empresas; esto implica velar por mejores estándares de seguridad para los trabajadores, ya sea a nivel individual como a nivel de procesos organizacionales. Dentro de los esquemas de trabajo que se utilizan para avalar este propósito, se encuentran como referencias las normas ISO y OHSAS, entre otras normativas y estándares de seguridad y calidad del trabajo.

En Chile, el 28 de Julio de 2005 comienza a regir la ley $\mathrm{N}^{\circ} 20.001$ dictada por el Ministerio del Trabajo y Previsión Social, la cual regula el peso máximo de carga humana. A partir de esto una empresa metalúrgica ligada a la minería metálica, de manera pionera en el país, como parte de su política de Seguridad y Salud Ocupacional, se propone evaluar el cumplimiento de ésta en sus procesos, con el objetivo de cumplir con la legislación y de asegurar el bienestar de sus trabajadores, previniendo la aparición de enfermedades ocupacionales asociadas al manejo manual de cargas, sobre todo cuando a la fecha han existido 3 trabajadores que reciben Pensión de Invalidez a causa de una enfermedad profesional de tipo músculo esquelética producto del trabajo.

Por otra parte, la Escuela de Terapia Ocupacional de la Universidad Mayor es la primera escuela del país que establece una alianza con una empresa tendiente a incorporar la Terapia Ocupacional al interior del equipo de prevención de riesgos, a través de sus estudiantes en práctica profesional. De esta manera, los estudiantes pueden aprender el ejercicio profesional en un contexto diferente al tradicional (área salud) y la empresa se ve beneficiada al incorporar a su equipo de trabajo a un profesional con conocimientos de salud y comportamiento humano, aportando de esta manera, una mirada mas integral del trabajador y de las condiciones que se presentan en un ambiente laboral.

A partir de lo anterior es que durante el año 2007, una docente guía en conjunto con ocho estudiantes de dicha casa de estudios, llevan a cabo un diagnóstico respecto del manejo manual de cargas en el área productiva y de servicios en la empresa, para determinar la magnitud de la situación y posibles intervenciones futuras.

\section{Ley Número $20.001^{1}$}

En Chile el año 2005 el Ministerio del Trabajo y Previsión Social promulga la ley que regula el peso máximo de carga humana, la cual incorpora en el libro II del Código del trabajo el Título V "De la Protección de los Trabajadores de Carga y Descarga de Manipulación Manual”, la cual esta basada en el Real Decreto 487/1997 desarrollado por el Instituto Nacional de Salud e Higiene en el Trabajo (INSHT, España) ${ }^{2}$, que 
además cuentan con una guía técnica de evaluación y prevención en este tema ${ }^{3}$.

Establece las normas que se aplican a las manipulaciones manuales que impliquen riesgo a la salud o a las condiciones físicas de los trabajadores, asociados a las características y condiciones de la carga. Para ello se entiende que la "manipulación comprende toda operación de transporte o sostén de carga cuyo levantamiento, colocación, empuje, tracción, porte o desplazamiento exija esfuerzo físico de uno o varios trabajadores”.[1, 2005]

Esta ley plantea 2 elementos centrales:

1.- La responsabilidad que recae en el empleador en velar por que existan los medios adecuados (especialmente mecánicos) para evitar la manipulación habitual de carga y procurar que el trabajador que realice este tipo de tareas reciba formación respecto de los métodos de trabajo que debe utilizar a fin de proteger su salud, además de evaluar y controlar los riesgos asociados a esta tarea.

2.- El peso máximo de manipulación no puede exceder los $50 \mathrm{Kg}$., la que en el caso de mujeres y menores de 18 años se reduce a $20 \mathrm{Kg}$. como máximo sin ayuda mecánica y prohíbe la manipulación de carga a mujeres embarazadas. Estos valores operan cuando la manipulación es inevitable, es decir cuando las "características del proceso no permiten utilizar medios o ayudas mecánicas”. [1, 2005]

\section{METODO}

El presente estudio, de tipo descriptivo, se basa en cuatro condiciones fundamentales:

- Presencia de 3 trabajadores acogidos a la ley 16.744 (Ministerio del trabajo 1968, Chile) que reciben Pensión de Invalidez, a causa de una enfermedad profesional de tipo músculo esquelética.

- Cumplir con la legislación y asegurar el bienestar de los trabajadores, previniendo la aparición de enfermedades ocupacionales asociadas al manejo manual de cargas.

- Necesidad de conocer con mayor precisión las condiciones y riesgos en la manipulación manual de carga, tarea desarrollada dentro de la empresa, a fin de saber el impacto que puede tener en la adquisición de enfermedades profesionales.

- Abordar la problemática del manejo manual desde una perspectiva más amplia que trascienda la mera medición cuantitativa de los riesgos a los cuales se ven expuestos los trabajadores. En ese entendido el estudio de puestos de trabajo, metodología utilizada ampliamente por los Terapeutas Ocupacionales, constituye un aporte significativo en cuanto a la evaluación y diseño de las condiciones de los puestos de trabajo y los elementos ambientales que se encuentran presentas. Por último la realización del estudio incorpora una mirada del trabajo desde la óptica de la salud.

\section{Objetivo general}

Conocer las condiciones en que se realiza el manejo manual de carga en las áreas productivas y de servicios en la empresa, con el fin de identificar y posteriormente 
proponer estrategias para controlar los factores asociados y que puedan ocasionar riegos de accidente o enfermedad al trabajador.

\section{Objetivos específicos}

1. Identificar los puestos de trabajo que en los cuales se realiza manejo manual de carga.

2. Identificar aquellos puestos de trabajo que representan un mayor riesgo en la salud de los trabajadores asociados al manejo manual de carga.

3. Identificar y jerarquizar los factores que influyen en la ejecución incorrecta manipulación de carga.

Se realizó entre los meses de Marzo a Septiembre del 2007, en los puestos de Trabajo de las áreas productivas, (pirometalúrgicas e hidrometalúrgicas) en donde el principal manejo manual de carga es el rodamiento de tambores y el área de servicio como (ej.: bodega, mantención mecánica), realizándose transporte de materiales, herramientas y equipos principalmente.

La metodología se basó en el análisis ocupacional ${ }^{4}$, utilizando el estudio de puesto de trabajo, el cual implica la identificación y descripción cualitativa de los elementos que intervienen en el puesto, independientemente del trabajador que lo ejecuta, con el fin de describir tareas y operaciones del puesto; describir patrones motores implícitos en su ejecución; determinar existencia o ausencia de factores de riesgo en la génesis de las molestias; corregir aspectos físicos y técnicas de trabajo no adecuadas. A partir

Además, se utilizó el Software Ergocargas desarrollado por la Asociación Chilena de Seguridad, ACHS que permite cuantificar el nivel de riesgos asociados a los puestos de trabajo.

Cabe mencionar, que este estudio utiliza como instrumentos de recolección de datos la Observación Participativa: que se refiere a la observación en terreno de la forma de proceso, en relación a la ejecución del puesto de trabajo, visualizando todos los elementos que se ven influenciados entre si al momento de desarrollar las tareas. [4, 1988]. Para ello se realizaron entrevistas a los jefes y trabajadores, a quienes antes de iniciarlas se les consulta sobre su interés en participar, es importante señalar que siempre existió una buena recepción por parte de todos los estamentos de la empresa.

Esta información fue registrada de manera audiovisual y en una pauta de estudio de puesto de trabajo que contiene la siguiente información: identificación del puesto de trabajo; resumen descriptivo que de la o las funciones principales con sus respectivas tareas, pasos y operaciones; requisitos de ejecución tales como responsabilidad, conocimiento del trabajo; requisitos de conocimientos tecnológicos (vocabulario técnicos, y de maquinarias), Factores ambientales (ubicación del trabajo y las condiciones de este); Ropa y elementos de seguridad; y Nivel de esfuerzo.

Respecto de la organización y análisis de la información obtenida a partir de Estudio de Puesto de trabajo, se realizaron dos procedimientos: 
Primero, se aplica el Software Ergocargas, que entrega como resultado el nivel de riesgo que presenta el puesto de trabajo, definidos de la siguiente manera:

Nivel Moderado: Auque no existe una situación de riesgo alto, es recomendable examinar la tarea cuidadosamente. ${ }^{5}$

Nivel Alto: Se requiere introducir mejoras pronto. Esta situación podría exponer a riesgo de lesiones a la espalda a una proporción significativa de trabajadores. [5,2007]

Nivel Muy Alto: La tarea evaluada podría representar riesgo serio de lesiones a la espalda, por lo que debería analizarse detenidamente para introducir mejoras.[5, 2007]

Cabe destacar que es un método de screening cuyo nivel de sensibilidad es de un $83 \%$ y su valor predictivo es de un 71\%. [5, 2007]

Segundo, se establecen categorías a los riesgos observados, para luego cuantificar la frecuencia de aparición. A continuación se describen cada una de ellas:

1. Inadecuado procedimiento del trabajador en el manejo manual de carga, el trabajador ejecuta el levantamiento o transporte sin llevar a cabo el procedimiento correcto para ello, como por ejemplo, flexiona tronco y no rodilla.

2. Inadecuado diseño y dimensiones de elementos de trabajo respecto de medidas antropométricas del trabajador, se refiere a que los elementos de trabajo, que incluyen las máquinas, herramientas o materiales, presentan un diseño inadecuado para su utilización, es decir, controles y mandos difíciles de accionar, o que la estructura global de ellos presentan dimensiones que hacen que el trabajador trabaje sobre el nivel de sus hombros o bajo nivel de cadera, situación que a largo plazo ocasiona lesiones músculo esqueléticas de tren superior y lumbar.

3. Desorganización del espacio y materiales, este aspecto incluye:

- El orden del lugar de trabajo, es decir que exista presencia de otros elementos ajenos a la ejecución de la tarea que obstaculicen y que puedan ser condiciones inseguras para el trabajador.

- Los elementos de trabajo necesarios para el desempeño se encuentran distantes unos de otros, lo cual hace en el caso de manipulación de carga el trabajador traslade la carga distancias mayores a las necesarias.

- Los elementos de trabajo se encuentran próximos, pero su distribución en el espacio dificultan el desempeño de la tarea.

4. Factores Ambientales, incluyen condiciones del espacio de trabajo que se relacionan con la iluminación, temperatura, ventilación, presencia de obstáculos, espacio de trabajo reducido, pisos mojados e irregulares.

5. Falta de herramientas y elementos de apoyo, tienen relación con la ausencia de 
máquinas, herramientas o materiales necesarios para facilitar las tareas específicas del puesto de trabajo y/o de transporte, tendientes a disminuir la fatiga simplificando el método de trabajo, por ejemplo, incorporar una báscula cercana al lugar de trabajo que se traduzca en que la carga se movilice menor distancia.

6. Inadecuado procedimiento en uso de herramientas o elementos de apoyo, se refiere a que si bien en el puesto de trabajo existen herramientas, máquinas o materiales que facilitan la tarea, el trabajador no las usa o las usa incorrectamente por desconocimiento e inexperiencia.

7. Falta de mantención de herramientas y elementos mecánicos, se refiere a que existen las herramientas, maquinarias y materiales que facilitan la manipulación de la carga, pero estos se encuentran en malas condiciones, ocasionando que el trabajador realice un mayor esfuerzo innecesariamente.

\section{RESULTADOS}

Se analizaron un total de 82 puestos de trabajo (PT) de los cuales un 70,7\% pertenecen a las plantas productivas y un $29,3 \%$ a las áreas de servicios.

Gráfico N¹ Puestos de Trabajo evaluados por área.

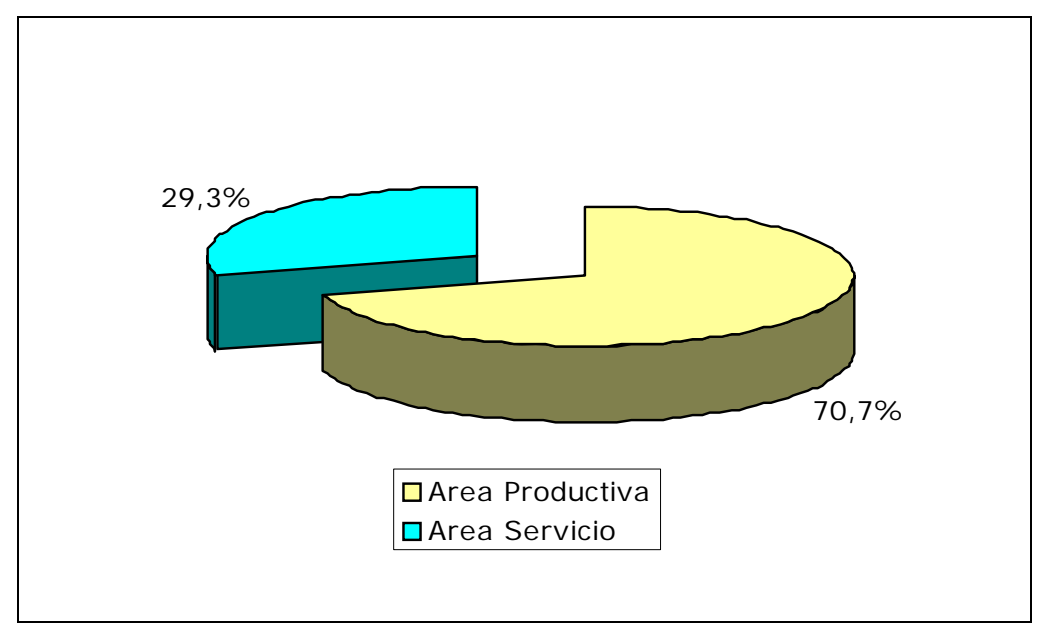

Del total de puestos evaluados, en un 54,9\% (45 PT) de ellos se realizan tareas asociadas al manejo manual de carga, y de ese porcentaje un 71,1\% (32 PT) pertenecen a área productivas y un 28,9\% a área de servicio (13 PT). 
Gráfico N² Total de Puestos de Trabajo por área que realizan o no Manejo Manual de Carga (MMC)

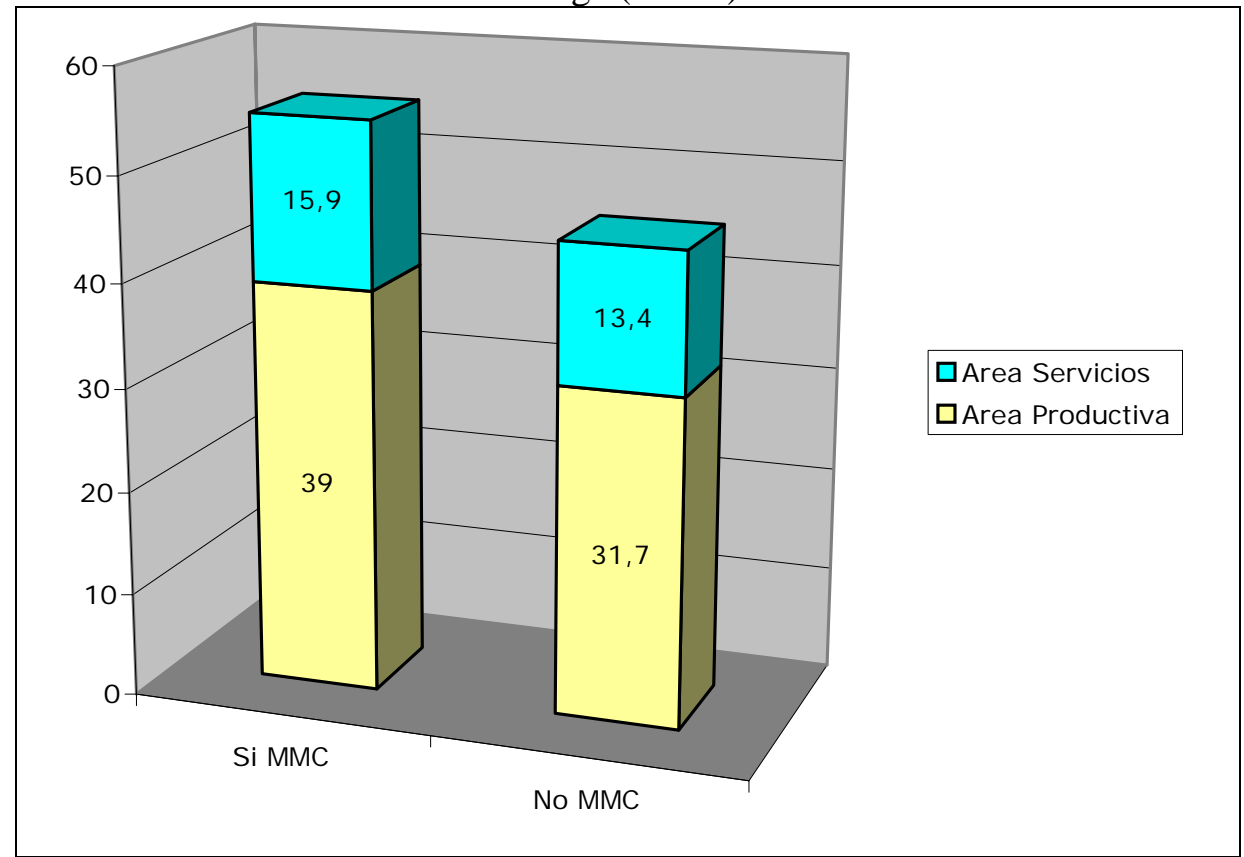

Al analizar las condiciones en que se realiza el MMC, se determina que en el $82,2 \%$ (37 PT) de los puestos de trabajo, requieren de la aplicación de medidas correctivas y el $17,8 \%$ (8 PT) no las requieren.

Cabe destacar, que la mayor parte de los puestos de trabajo que realizan MMC y que requieren de medidas correctivas pertenecen a las áreas productivas (53,3\% área productiva (24 PT) y 28,9\% (13 PT) área servicio).

Por otra parte, el total de los puestos de trabajo del área de servicio que realizan MMC requieren de la aplicación de medidas correctivas, es decir que presentan factores que pueden ocasionar daño en la salud de los trabajadores producto de las condiciones en que realizan esta tarea y que deben ser controlados. 
Gráfico N³: Porcentaje de Puestos de Trabajo que requieren la aplicación de medidas correctiva por área de la empresa

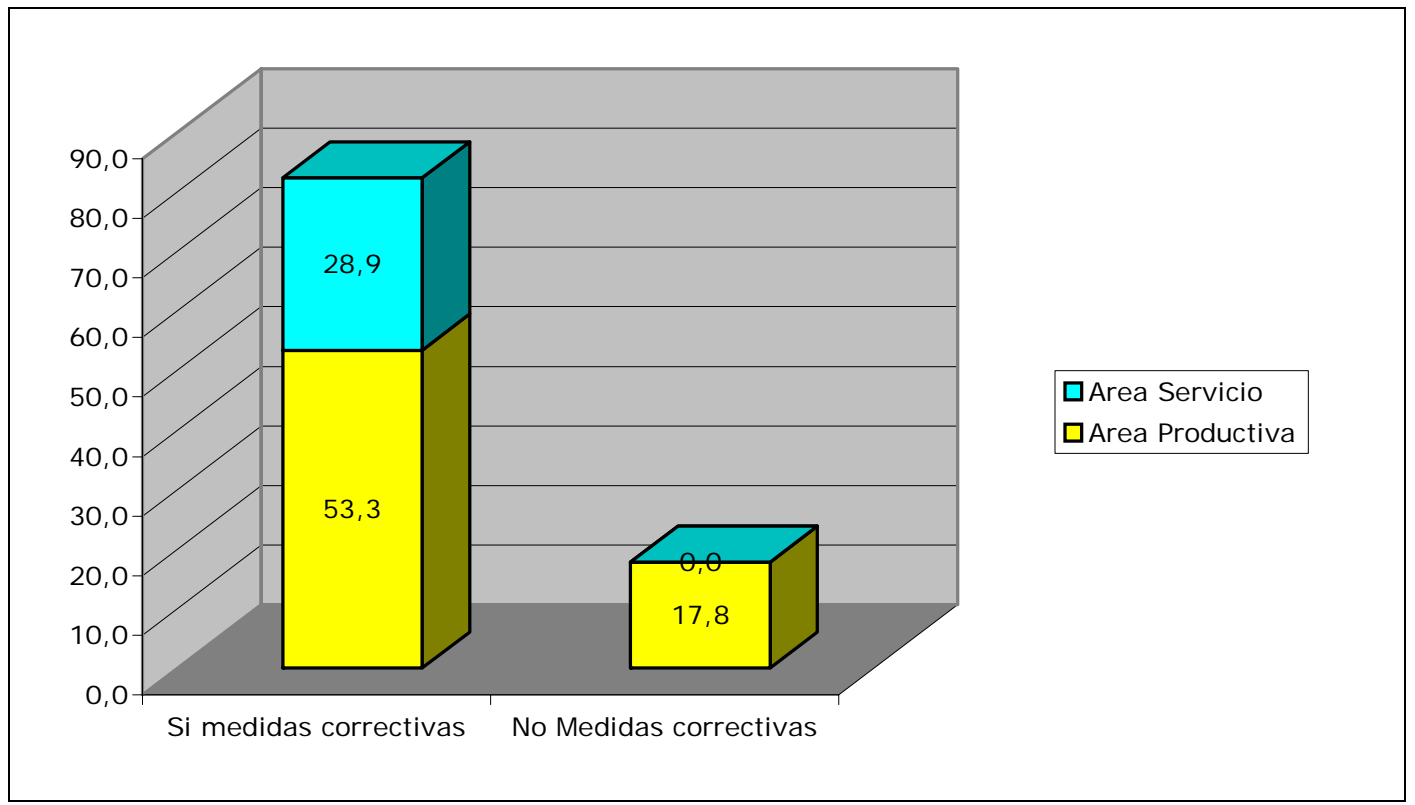

A los puestos de trabajo que incluye dentro de sus funciones el manejo manual de carga y que requieren medidas correctivas (37 PT), se aplicó el Software Ergocargas para cuantificar el nivel de riesgo que presentan para la salud de los trabajadores.

Se obtuvo como resultado que el 56,7\% (21 PT) del total de puestos de trabajo que realizan Manejo Manual de carga (37 PT) presentan riesgo Moderado y se concentran principalmente en aquellos pertenecientes a las área productiva, el 35,1\% (13 PT) riesgo Alto y el 8,1\% (3 PT) riesgo Muy Alto, estos últimos se distribuyen de manera similar en ambas áreas (productivas y servicio).

Gráfico $\mathrm{N}^{\circ} 4$ : Nivel de Riesgo presente en los puestos de trabajo en que se realiza el MMC y que requieren de medidas correctivas.

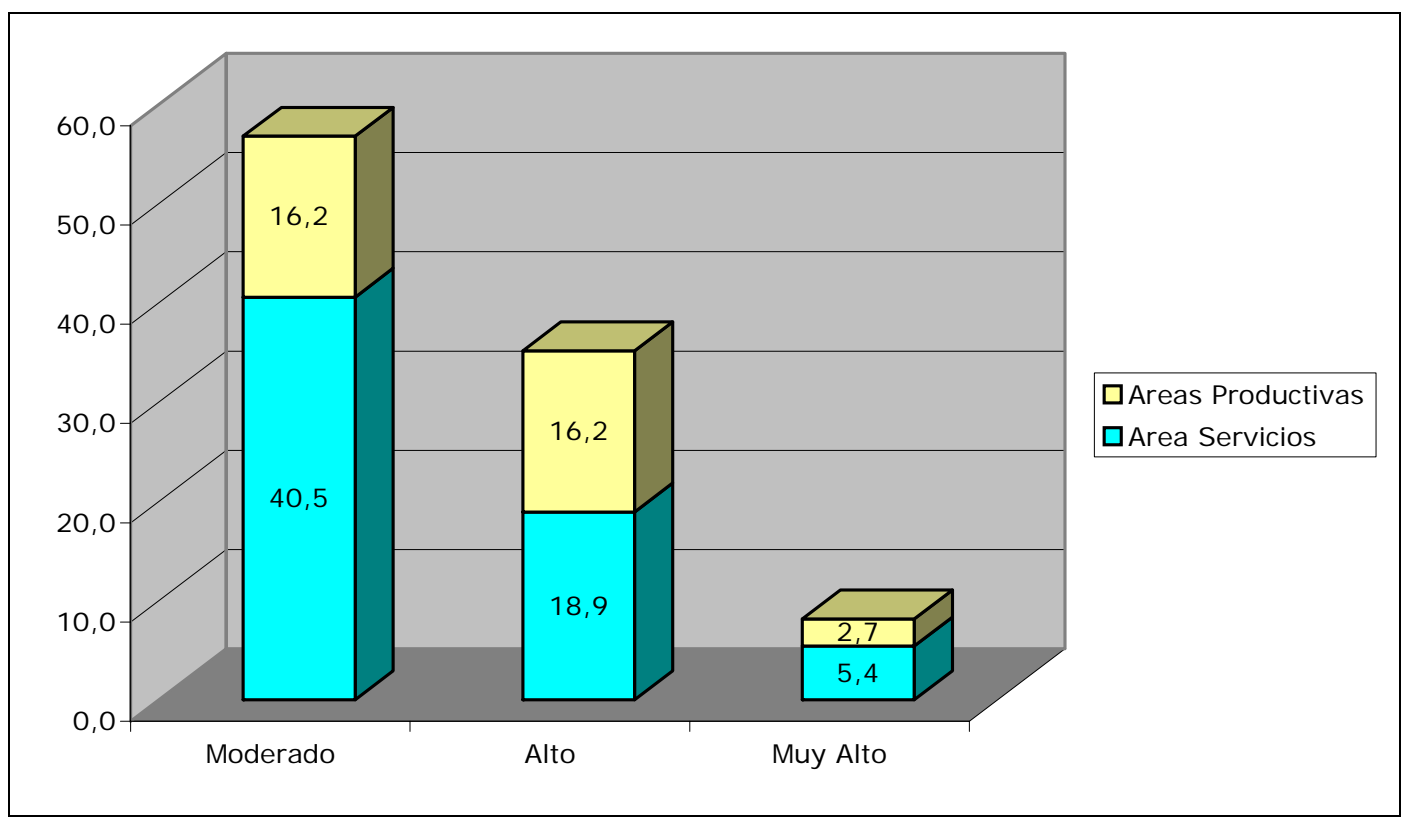


Respecto de las categorías de análisis de los factores de riegos presentes en los puestos de trabajo, definidas a partir de la aplicación del Estudio de Puesto de Trabajo, se determinó que de los siete factores estudiados (descritos en la metodología), se presentan con mayor frecuencia (20.63\%), los Factores Ambientales, principalmente relacionados con ventilación, iluminación, presencia de obstáculos en lugares de tránsito, espacios de trabajo reducido y pisos mojados e irregulares.

En segundo lugar con un 17,46\% se encuentra el inadecuado procedimiento que realiza el trabajador en el manejo manual de carga, seguido con un $16,67 \%$ la falta de herramientas y elementos de apoyo en la ejecución del trabajo, que disminuyan la fatiga, simplificando el método de trabajo.

Gráfico N5: Factores de riesgos asociados al manejo manual de carga según Estudio de Puesto de Trabajo

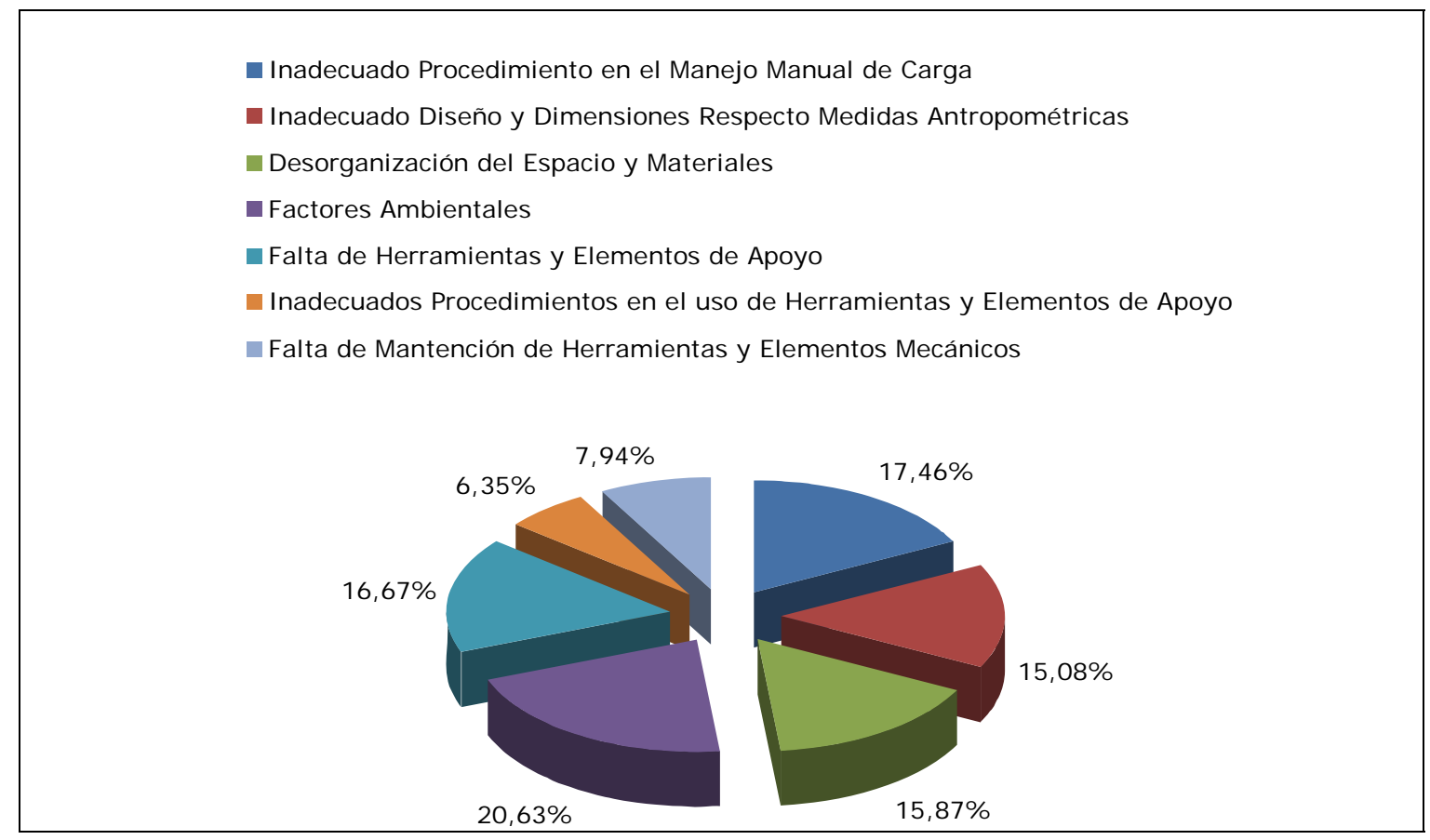

\section{DISCUSION}

A partir de los resultados obtenidos se puede establecer la importancia que tiene realizar este tipo de estudio, ya que permite conocer las características de la manipulación de carga, atingentes a la realidad de cada empresa, determinar los factores de riesgo involucrados en ella, constituyendo una herramienta fundamental para diseñar programas que incorporen las acciones correctivas necesarias, con la finalidad de asegurar la salud ocupacional, evitar accidentes y enfermedades profesionales (lesiones músculo esqueléticas) a largo plazo asociadas al manejo manual de cargas. ${ }^{6} 78$ 
Por otra parte se evidencia la importancia que ha tenido la incorporación de Terapeutas Ocupacionales en el área de Prevención de Riesgos y en particular en el diagnóstico de los factores de riesgos asociados al manejo manual de carga, ya que permitió observar la problemática desde miradas diferente y complementaria, entre profesionales de la salud y de la ingeniería.

Sobre todo, cuando la Terapia Ocupacional centra su intervención en las ocupaciones humanas, siendo el trabajo una de ellas, tal como lo define la Asociación Americana de Terapia Ocupacional ${ }^{9}$ [9], y por consiguiente, están interactuado las características del cliente, el contexto y sin duda los patrones del desempeño, ejes de nuestro quehacer profesional.

Si se analizan los resultados obtenidos, desde el punto de vista de la Terapia Ocupacional, claramente los factores de riesgos identificados en el manejo manual de carga, se relacionan con el contexto, fundamentalmente físico y cultural, y sobre todo con los hábitos y rutinas que los trabajadores tienen en la ejecución de su trabajo y en particular en el manejo manual de carga.

Se confirma la multiplicidad de factores que están presentes en el manejo manual de carga, los cuales interfieren de forma negativa en las condiciones de salud del trabajador, y que su control involucra a la organización en su conjunto, requiriendo del compromiso tanto de los directivos como de sus trabajadores.

La intervención, debe estar encaminada a generar cambios en el desempeño ocupacional de los distintos integrantes de la empresa, de manera de prevenir la aparición de enfermedades o discapacidades, de favorecer el bienestar y la satisfacción vital del trabajador. Propósitos fundamentales de la Terapia Ocupacional al intervenir en las diversas ocupaciones cotidianas en que participa el ser humano.

No se debe olvidar, que la visión de la Terapia Ocupacional debe ir en paralelo con los objetivos empresariales, los cuales están dirigidos esencialmente a la eficiencia productiva. Por lo tanto, las estrategias desarrollar constituyen un gran desafío para el Terapeuta Ocupacional.

El presente trabajo incorporó metodologías de evaluación de carácter cuantitativa (Ergocargas) y cualitativa (Estudio de Puesto de Trabajo), comprobándose la relevancia que tuvo esta estrategia, ya que cada una por sí sola es insuficiente para observar y conocer la realidad del manejo manual de carga.

En este caso el software Ergocargas permitió cuantificar el nivel de riesgos, información fundamental a la hora de planificar y orientar la estrategia de intervención, que en primera instancia debiese abordar aquellos puestos de trabajo o factores que presenten mayor riesgo a los trabajadores. Por otra parte el Estudio de Puesto de trabajo, permite identificar con mayor precisión los factores de riesgos involucrados, de tal manera que el diseño de las soluciones se ajusten a las características de los trabajadores, productivas, organizacionales y ambientales presentes.

Dentro de los factores de riesgos con mayor prevalencia se encontraron aquellos asociados a las condiciones ambientales y al inadecuado procedimiento en el manejo manual de carga realizado por los trabajadores. A partir de ello se desprende que: 
1.- En el primer caso, cabe destacar que para su abordaje, se requiere que la empresa dentro de su programa de construcción, remodelación y ampliación incorpore estos elementos, esta manera se previenen los factores de riesgos, lo cual implica un menor costo, teniendo presente que es mas económico construir bien desde un principio que hacer arreglos posteriores.

2.- En el segundo caso, la capacitación constituye una herramienta fundamental para su control, ratificando lo estipulado por la ley $\mathrm{N}^{\circ} 20.001$, siempre y cuando la metodología usada sea coherente con las características de los trabajadores y la organización, de manera que implique un aprendizaje significativo y en última instancia un cambio de conducta.

La ley º20.001 estipula la incorporación de herramienta mecánicas para evitar la manipulación habitual de carga, sin embargo, para que estas puedan cumplir su función correctamente, debe existir dentro de la empresa un programa de mantención, principalmente de carácter preventivo, porque de lo contrario no se resuelve el problema, sino se agrava, haciendo que el trabajador realice un mayor esfuerzo físico al utilizar una herramienta en mal estado o simplemente porque las deja de utilizar.

Por otra parte, dicha ley se hace insuficiente en su planteamiento, en tanto estipula la disminución de la manipulación de carga al incorporar elementos mecánicos, el peso máximo y la implementación de capacitación, dejando de lado las condiciones que se debe tener cuando la manipulación de carga sea inevitable. Es decir, no se describen las características de tamaño, agarre, frecuencia y los factores ambientales, que en el caso de este estudio constituye el factor que se presenta con mayor frecuencia, para un manejo manual de carga seguro para la salud del trabajador.

La observación directa de la ejecución de las tareas y sus respectivas condiciones, constituyó un elemento central en el presente estudio, a partir de la cual, se determinó que el control de la mayor parte de los factores de riesgo se puede hacer con la aplicación de medidas simples que incluso no requieren de recurso económico significativo, como por ejemplo el orden de los elementos de trabajo.

Finalmente, garantizar que la manipulación de carga implique el menor riesgo para los trabajadores, pasa por comprender que es un tema transversal, que involucra a las distintas áreas de una empresa, tales como el área de Recursos Humanos, de Prevención de Riesgos, de Desarrollo de proyectos, de finanzas, abastecimiento, etc. que den cuenta de una política común al respecto.

\section{REFERENCIAS BIBLIOGRAFICAS}

\footnotetext{
${ }^{1}$ Ministerio del Trabajo y Previsión Social, LEY N² 20.001: "Regula Peso Máximo de Carga Humana”, disponible en www.bcn.cl/lc/bleyes/, marzo 2005

${ }^{2}$ Ministerio del Trabajo y Asuntos Sociales de España, Real Decreto REAL DECRETO 487/1997, del 14 de abril, sobre disposiciones mínimas de seguridad y salud relativas a la manipulación manual de cargas que entrañe riesgos, en particular dorsolumbares, para los trabajadores. Disponible en www.insht.es/InshtWeb/Contenidos/Normativa/TextosLegales/RD/1997/487_97/PDFs/ realdecreto4871997de14deabrilsobredisposicionesminimasd.pdf. marzo 2007.
} 
${ }^{3}$ Instituto Nacional de Seguridad e Higiene en el Trabajo e Inmigración, Guía Técnica para la Evaluación y Prevención de los Riesgos Relativos a la Manipulación Manual de Carga, España, Ministerio del Trabajo 2003

${ }^{4}$ VERGARA, C., (1988); Curso de Análisis Ocupacional. Venezuela: Glarp, 1988; 5595.

${ }^{5}$ EYQUEN, L,; CÓRDOVA, V.; MALDONADO, O., Usabilidad, Confiabilidad y validez de un Método para la Evaluación de Factores de Riesgo en el Manejo Manual de Cargas. Revista “Ciencia y Trabajo”, de la Fundación Científica y Tecnológica ACHS 2007; 9-25. Disponible en www.cienciaytrabajo.cl | AÑO 9 | NÚMERO 25 | JULIO / SEPTIEMBRE 2007 |.

${ }^{6}$ CHAMBY ,V. , Evaluación y Control en la Manipulación Manual de cargas. 2007, disponible en www.monografias.com/trabajos43/manipulacion-cargas/manipulacioncargas.shtml?monosearch

${ }^{7}$ Agencia Europea para la Seguridad y la Salud en el Trabajo, Riesgos Asociados a la Manipulación Manual de Cargas en el Lugar de Trabajo, España 2007. Disponible en http://osha.europa.eu/es/publications/factsheets/73. Marzo 2007

${ }^{8}$ Federación de Empresas de la Rioja; Manipulación Manual de Carga, Argentina. Disponible en http://sie.fer.es/esp/asesorias/riesgos/Biblioteca/SECTORIALES/Manipulacion_manual _cargas/webDoc_9031.htm. Marzo 2007

${ }^{9}$ Asociación Americana de Terapia Ocupacional, Occupational Therapy Practice Framework: Domain and Process. Revista The American Journal of Occupactional Therapy, Noviembre/Diciembre 2002 volumen 56 Número 6, 609-639. 